\title{
Full band quantum transport of heterojunction electron devices with empirical pseudopotentials
}

\author{
Adel M'foukh, Marco G. Pala Member, IEEE, David Esseni Fellow, IEEE
}

\begin{abstract}
This paper presents the methodology, implementation and application of a full-band quantum transport model based on the non-equilibrium Green's function formalism and the empirical pseudopotentials. In particular the paper reports the treatment of heterojunctions between lattice matched semiconductors, comprising a gradual transition region described according to a virtual crystal approximation. Our approach entails several numerical techniques to make the full-band quantum transport method computationally affordable, and thus enable robust and efficient self-consistent device simulations.

Then we employ our simulation scheme for the analysis of some exemplary devices based on quantum tunnelling, such as an Esaki tunnelling diode, as well as $n$ - and $p$-type heterojunction Tunnel FETs. In particular we investigate the influence on the current-voltage characteristics of the width of the heterojunction transition region. We observe that a gradual transition region mainly affects the device characteristics by lengthening the tunnelling path at the heterojunction, which has a different impact on device current depending on the external bias conditions.
\end{abstract}

Index Terms-Empirical pseudopotential, quantum transport, NEGF, heterojunctions, electronic devices.

\section{INTRODUCTION}

$\mathbf{T}$ HE aggressive shrinkage of FETs has effectively turned them into tiny quantum constrictions where quantum transport effects have become important, such as the sourcedrain tunnelling in MOSFETs [1], [2], or the band-to-bandtunnelling (BTBT) in Tunnel FETs [3], [4]. The interface to volume ratio has also steadily increased in $3 \mathrm{D}$ device architectures, culminating in CMOS stacked nanowire transistors [5], [6], [7], [8].

The use of heterojunctions is also an important technology option used, among other possible examples, in bipolar transistors [9][10], as well as in Tunnel FETs [11], [12]. Moreover there is clear experimental evidence that fabricated heterojunctions are not abrupt, but instead feature a transition region extending for a few atomic layers [13][14][15], which solicits an atomistic description of the interfaces.

Actually an atomistic approach is nowadays perceived as the frontier of transport modelling, and different efforts have been reported for models based directly on first-principle calculations, which in turn have become available in several ab-initio packages [16], [17].

An empirical pseudopotentials method (EPM) provides an interesting compromise between accuracy and computational burden, in that it can accurately and effectively describe both

A. M'foukh and M. G. Pala are with Université Paris-Saclay, CNRS, Centre de Nanosciences et de Nanotechnologies, 10 Bd Thomas Gobert, 91120 Palaiseau, France

D. Esseni is with DPIA, University of Udine, Via delle Scienze 206, 33100 Udine, Italy bulk and nanostructured semiconductors by using only few fitting parameters [18], [19], [20], [21], [22]. Some recent papers have explored the use of the EPM for transport modelling by using either a quantum transmitting boundary method [23], [24], [25], [26], or a Non Equilibrium Green's Function (NEGF) method [27], [28], [29]. However, no attempts have been reported to our knowledge for the use of such transport methods for electron devices whose operation relies on the heterojunction of different semiconductors.

In this work we report a substantial extension of our recent work related to EPM based quantum transport, and we describe the treatment of lattice matched heterojunctions in nanoscale devices. We here propose a methodology for the description of either abrupt or gradual heterojunctions, where the EPM parameters are changed with the position so as to ensure a correct alignment of conduction and valence band edges along the heterojunction. The NEGF based formalism for quantum transport is also extended in order to duly account for a non homogeneus system. Two exemplary and yet technologically relevant case studies are then addressed, namely a III-V semiconductor based Esaki diode and both $n$ - and $p$-type heterojunction Tunnel FETs.

The paper is organized as follow. Section II illustrates the self-consistent transport model by focussing on the treatment of heterojunction within the EPM method. In Sec. III we report some simulation case studies for devices employing heterojunctions, and in Sec. IV we finally offer our concluding remarks.

\section{MODEL DESCRIPTION}

Here we briefly describe the full-band model employed in this work for band-structure and quantum transport calculations, which is based on the empirical pseudopotential method (EPM) [30], [31]. The general methodology has been already described in previous publications [27], [28], so that we here focus on the aspects related to the treatment of heterojunctions that have been explicitly developed for this work.

We used a local formulation of the EPM where, for a diamond semiconductor (e.g. Si or Ge) or a zinc-blende compound (e.g. GaAs, InAs), the spectral components of the pseudopotential can be written as [30], [31]

$$
V_{L}(\mathbf{G})=U_{S}(|\mathbf{G}|) \cos [\mathbf{G} \cdot \boldsymbol{\tau}]+i U_{A}(|\mathbf{G}|) \sin [\mathbf{G} \cdot \boldsymbol{\tau}]
$$

where $\mathbf{G}$ is a reciprocal lattice vector and $\boldsymbol{\tau}=(1 / 8) a_{0}(1,1,1)$ is the atomic basis vector. The material dependent $U_{S}(|\mathbf{G}|)$, $U_{A}(|\mathbf{G}|)$ denote respectively the symmetric and antisymmetric form factors, with $U_{A}(|\mathbf{G}|)$ being null for diamond materials. It is well known that the full band relation of most technologically relevant semiconductors can be described well by 
using only three non null $U_{S}(|\mathbf{G}|)$ and $U_{A}(|\mathbf{G}|)$ form factors, corresponding to $|\mathbf{G}|^{2}=3,8,11\left[2 \pi / a_{0}\right]^{2}$ for $U_{S}(|\mathbf{G}|)$ and to $|\mathbf{G}|^{2}=3,4,11\left[2 \pi / a_{0}\right]^{2}$ for $U_{A}\left(|\mathbf{G}|\right.$ ) (with $a_{0}$ being the lattice constant) [30].

The quantum transport model used in this work relies on the Non Equilibrium Green's Function (NEGF) method formulated in a hybrid basis, $x \mathbf{K}_{y z}$, consisting of a discretized real space in the transport direction $x$ and of plane waves in the $(y, z)$ directions [28]. In the hybrid basis the Hamiltonian matrix inside a given semiconductor takes the block tridiagonal form

$\left[\begin{array}{ccccccc}\mathbf{H}\left(x_{1}, x_{1}\right) & \mathbf{H}_{0,1} & 0 & 0 & \ldots & & \\ \mathbf{H}_{0,1}^{\dagger} & \mathbf{H}\left(x_{2}, x_{2}\right) & \mathbf{H}_{0,1} & 0 & \ldots & \\ \ldots & & \ddots & & & \\ 0 & 0 & \mathbf{H}_{0,1}^{\dagger} & \mathbf{H}\left(x_{j}, x_{j}\right) & \mathbf{H}_{0,1} & 0 & \ldots \\ & & \ldots & \ldots & \ldots & & \end{array}\right]$

where $x_{j}$ is a discretization point in the transport direction. For a 3D electron gas (i.e. no quantum confinement in directions $y, z)$ the size of each blocks in Eq. (2) is $M_{3 D}=2 N_{G} / N_{d}$, where $N_{G}$ is the number of $\mathbf{G}$ vectors in EPM calculations (which is in turn set by the EPM cutoff energy), and $N_{d}$ is the number of discretization points inside a unit cell. For a 2D or a 1D electron gas the Hamiltonian matrix still has the tridiagonal form in Eq. (2), but the size of the blocks is increased [28]. In all the calculations of this work we used $N_{d}=32$, and an EPM cutoff energy $E_{c u t}=4.5$ Ry corresponding to $N_{G} \approx 800$.

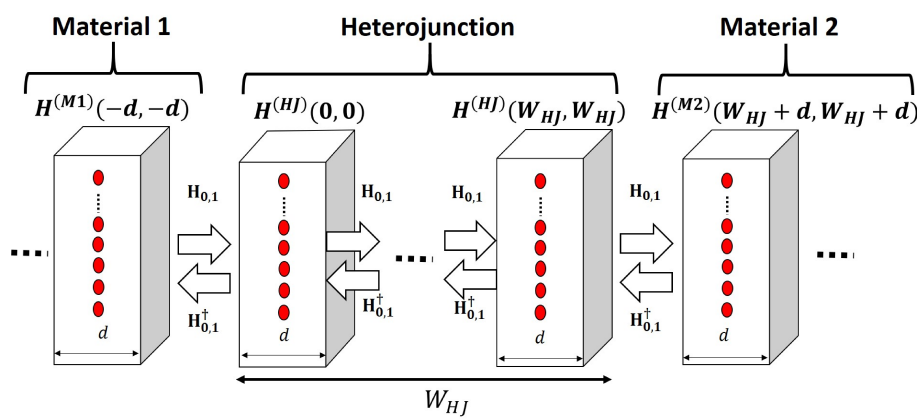

Fig. 1. Pictorial illustration of the blocks of the Hamiltonian matrix across a gradual heterojunction where, in order to simplify the notation, one end of the heterojunction has been placed at $x=0 . W_{H J}$ is the width of the heterojunction transition region and $d=a_{0} / N_{d}$ is the discretization step along $x$. The notation is consistent with Eq.2. In the heterojunction region the EPM parameters change with the position and thus contribute to the position dependence of the diagonal Hamiltonian blocks, $H^{H J}\left(x_{i}, x_{i}\right)$.

\section{A. Modelling of heterojunctions with the EPM method}

For a homogeneous semiconductor the diagonal blocks $\mathbf{H}\left(x_{j}, x_{j}\right)$ in Eq. (2) depend on $x_{j}$ only through the local pseudopotential $V_{L}\left(x_{j}, \mathbf{G}_{y z}-\mathbf{G}_{y z}^{\prime}\right)$, which is obtained via a discrete Fourier transform from the $G_{x}$ to the $x_{j}$ and it is periodic of $a_{0}$ [28]. In this work we studied lattice matched heterojunctions by using a virtual crystal approximation [32], whose soundness has been proved also for quaternary alloys [33]. In this approach the EPM parameters $U_{S}(|\mathbf{G}|), U_{A}(|\mathbf{G}|)$ change along the transport direction so as to describe either an abrupt heterojunction, or a gradual heterojunction having a transition width, $W_{H J}$, long up to a few lattice constants [34]. More precisely, the Hamiltonian blocks of the two materials are first computed in the hybrid space $x \mathbf{K}_{y z}$, then they are patched at adjacent positions to form the heterojunction. This approach implies that at the heterojunction interface a discontinuity exists in the empirical pseudopotential. For the case at study in this paper, however, such a discontinuity is small because $\mathrm{GaSb}$ and InAs have similar EPM spectral components (see Tab. I) The coupling Hamiltonian matrix between adjacent blocks is simply given by the spatial discretization of the kinetic energy operator [28], hence it is material independent.

TABLE I

EPM PARAMETERS IN RY FOR GASB AND INAS EMPLOYED IN THIS WORK. $U_{S}(0)$ IS SET TO 0 FOR INAS AND TO 0.84 EV FOR GASB.

\begin{tabular}{|c|c|c|c|c|c|c|}
\hline \hline & $U_{S}(3)$ & $U_{S}(8)$ & $U_{S}(11)$ & $U_{A}(3)$ & $U_{A}(4)$ & $U_{A}(11)$ \\
\hline $\mathrm{GaSb}$ & -0.22 & 0 & 0.05 & 0.06 & 0.05 & 0.01 \\
$\mathrm{InAs}$ & -0.22 & 0 & 0.05 & 0.08 & 0.05 & 0.03 \\
\hline \hline
\end{tabular}

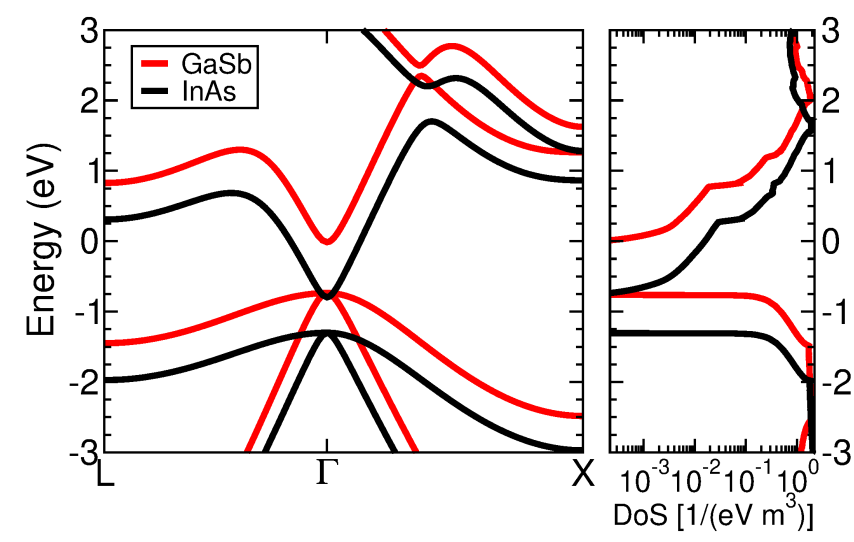

Fig. 2. (Left) Highest valance bands and lowest conduction bands for $\mathrm{GaSb}$ and InAs along the main symmetry points inside the first Brillouin zone and (right) the corresponding density of states.

In the description of heterojunctions via the EPM method the correct alignment of conduction and valence band edges between the different materials must be ensured. At this regard, Eq. (1) shows that a non zero $U_{S}(0)=U_{S}(|\mathbf{G}|=0)$ simply results in a rigid shift of $V_{L}$ and thus of the bandstructure of a given material. Consequently, in the analysis of a homogeneous system one typically sets $U_{S}(0)=0$, because $U_{S}(0)$ can be absorbed in the choice of the energy reference.

In our modelling of heterojunctions, however, $U_{S}(0)$ has been changed in order to ensure the correct alignment of the conduction and valence band edges of the constituent materials, which is an approach already discussed and validated in [35]. For devices including heterojunctions the position dependence of the EPM parameters contributes to the $x_{j}$ dependence of the diagonal Hamiltonian blocks $\mathbf{H}\left(x_{j}, x_{j}\right)$ in Eq. (2), as it is illustrated in the sketch of Fig. 1.

Tab. I reports the EPM parameters for GaSb and InAs used in all the calculations of this work, and Fig. 2 illustrates 


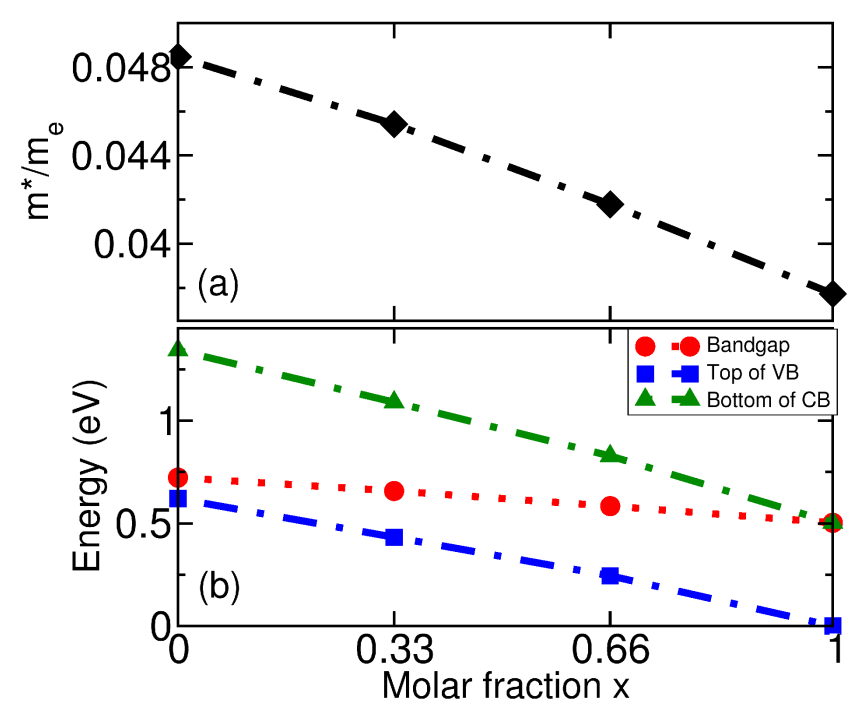

Fig. 3. (a) Electron effective mass $m^{*}$ at the $\Gamma$ minimum point of the conduction band versus the molar fraction $x$ of the alloy $\mathrm{GaSb}_{1-x} \operatorname{InAs} x$. (b) Top of valence band, bottom of conduction band and band-gap versus the molar fraction. The energy reference is the top of the InAs valence band.

the bandstructure of the two materials, as well as the corresponding density of states. As it can be seen, the GaSbInAs heterojunction provides a broken bandgap system, in the sense that the bottom of the InAs conduction band is slightly below the top of the $\mathrm{GaSb}$ valence band. By varying the molar fraction $x$ in $\mathrm{GaSb}_{1-x} \mathrm{InAs}_{x}$ the corresponding effective mass at the $\Gamma$ minimum of the conduction band change as reported in Fig. 3

\section{B. Quantum transport}

Our transport model is based on the NEGF method, where the transport equations are self-consistently solved with the Poisson equation for the overall device electrostatics. For ballistic transport the retarded Green's function can be formally defined as $\mathbf{G}_{r}(E)=\left[E \mathbf{I}-\mathbf{H}-\boldsymbol{\Sigma}_{L}-\boldsymbol{\Sigma}_{R}\right]^{-1}$, where $\mathbf{H}$ is the Hamiltonian matrix in Eq. (2), and $\boldsymbol{\Sigma}_{R / L}$ are the left and right contact self-energies. Because only a small subset of the blocks of $\mathbf{G}_{r}(E)$ are needed to calculate the carrier density and the current, we here employed the recursive Green's function algorithms based on Dysons's equations [36], which make the size of the Hamiltonian blocks the crucial parameter for the computational burden.

In this latter respect, we could obtain a substantial reduction of the size of such blocks by employing a mode-space transformation [37]. Differently from Ref.[28], the modes along the heterojunction direction change with the position $x_{j}$, which we duly accounted for in the transformation of the Hamiltonian to the mode space.

In the case studies of this work we found that, by selecting only the lowest energy transverse modes in each device section, the size of the Hamitonian blocks could be vastly reduced compared to the blocks in Eq. (2). Even in heterojunction devices, in fact, a few tens of modes were sufficient to achieve results that agree within a few percent with those obtained without introducing the mode space reduction.

\section{SELF-CONSISTENT DEVICE SIMULATIONS}

In this section we report exemplary simulation results obtained with the EPM based simulation methodology developed in this work for two band-to-band-tunnelling (BTBT) based devices employing a GaSb-InAs heterojunction. In all simulations the NEGF transport equations were solved selfconsistently with the Poisson equation describing the device electrostatics. All simulations were carried at room temperature and for the transport direction [100].
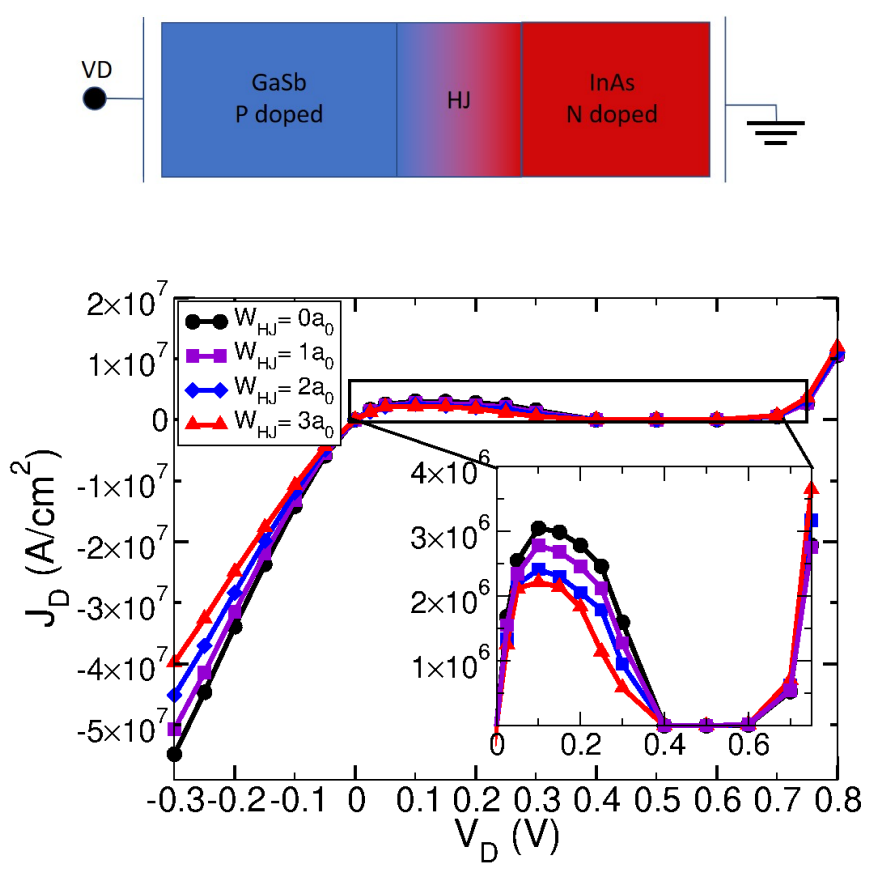

Fig. 4. (Top) Sketch of the Esaki diode where red is used for InAs and blue is used for GaSb, and (bottom) corresponding current density, $J_{D}$, versus the applied bias $V_{D}$, for different widths, $W_{H J}$, of the heterojunction transition region. The inset shows a focus on the negative differential resistance region.

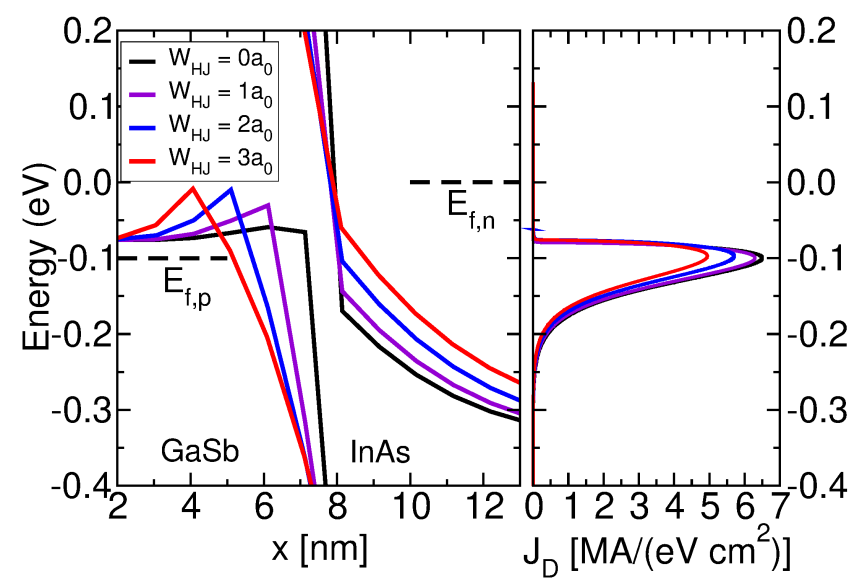

Fig. 5. (Left) Conduction and valence band profile along the Esaki diode for different widths $W_{H J}$ of heterojunction transition region. The source Fermi level, $E_{f, n}$ in the $n$ region is taken as the energy reference. (Right) Corresponding current spectrum at $k_{y}=k_{z}=0, J_{D}$, versus the energy and for different $W_{H J}$. The bias is $V_{D}=0.1 \mathrm{~V}$ 


\section{A. Esaki tunnelling diode}

The Esaki diode is sketched in Fig. 4 and consists of a $p$ type GaSb region and an $n$-type InAs region. The doping of the $p$ and $n$-type regions was set to respectively $N_{A}=10^{20} \mathrm{~cm}^{-3}$ and $N_{D}=3 \times 10^{19} \mathrm{~cm}^{-3}$, which result in a degenerate carrier gas in both regions. We assumed periodic boundary conditions along the transverse directions and sampled the wave vectors $k_{y}$ and $k_{z}$ with a constant step of $\Delta k_{y}=\Delta k_{z}=0.052 \pi / a_{0}$.

The current per unit area, $I_{D}$, versus the applied bias $V_{D}$ of the Esaki diode is shown Fig. 4 for different widths, $W_{H J}$, of the heterojunction transition region. In the forward bias a negative differential resistance (NDR) is observed, which is more clearly illustrated in the inset of the figure. As it can be seen the peak current in the NDR region decreases for more gradual heterojunctions (i.e. for larger $W_{H J}$ ), and it is maximum for the abrupt case. A similar dependence of $I_{D}$ on $W_{H J}$ is observed also in the reverse bias region, where the current is still completely dominated by BTBT in the bias range explored in Fig. 4.

Fig. 5 illustrates the conduction and valence band profiles along the device for $V_{D}=0.1 \mathrm{~V}$ (i.e. approximately at the peak current in the NDR region), as well as the energy resolved current density $J_{D}$. As expected the $J_{D}$ is practically relevant only in the narrow Fermi energy window identified by the Fermi levels $E_{f, n}$ and $E_{f, p}$ of respectively the $n$ - and $p$ region. Moreover, the narrower the transition region $W_{H J}$ is the shorter is the BTBT path, which in turn leads to the corresponding $I_{D}$ increase in Fig. 4.

TABLE II

DEVICE PARAMETERS FOR THE HETEROJUNCTION $n$-TYPE TUNNEL FET SIMULATED IN Fig. 6. $T_{c h}, L_{G}, L_{S / D}, N_{A}, N_{D}$ DENOTE THE CHANNEL THICKNESS, THE GATE LENGTH, THE SOURCE/DRAIN LENGTH AND THE DOPANT CONCENTRATIONS IN THE SOURCE AND DRAIN REGION, RESPECTIVELY.

\begin{tabular}{|c|c|c|c|c|c|}
\hline \hline & $\begin{array}{c}T_{c h} \\
{[\mathrm{~nm}]}\end{array}$ & $\begin{array}{c}L_{G} \\
{[\mathrm{~nm}]}\end{array}$ & $\begin{array}{c}L_{S / D} \\
{[\mathrm{~nm}]}\end{array}$ & $\begin{array}{c}N_{A} \\
{\left[\mathrm{~cm}^{-3}\right]}\end{array}$ & $\begin{array}{c}N_{D} \\
{\left[\mathrm{~cm}^{-3}\right]}\end{array}$ \\
\hline GaSb/InAs & 3.0 & 16 & 14 & $10^{20}$ & $10^{19}$ \\
\hline \hline
\end{tabular}

\section{B. Ultra-thin body, heterojunction Tunnel FETs}

Nanoscale FETs are often fabricated by using ultra-thin semiconductor films or nanowires, which can induce significant quantum confinement effects in the resulting low dimensional electron gas, as it is the case for the Tunnel FETs (TFETs) simulated in this section. The modelling of quantum confinement in our EPM based formalism has been described in Sec.II.A of reference [28], and it is based on a pseudo-oxide material. The EPM parameters of the pseudooxide are tailored so as to obtain the appropriate discontinuity at the semiconductor-oxide interface of the conduction band minimum and valence band maximum, which in turn confine the carriers in the semiconductor region. For both the $n$ - and the $p$-type TFETs we used a physical oxide thickness of $1.5 \mathrm{~nm}$ and a relative dielectric constant $\kappa=10$, corresponding to an equivalent oxide thickness of $0.6 \mathrm{~nm}$. The transverse wave vector $k_{y}$ was sampled with a constant step of $\Delta k_{y}=0.052 \pi / a_{0}$.
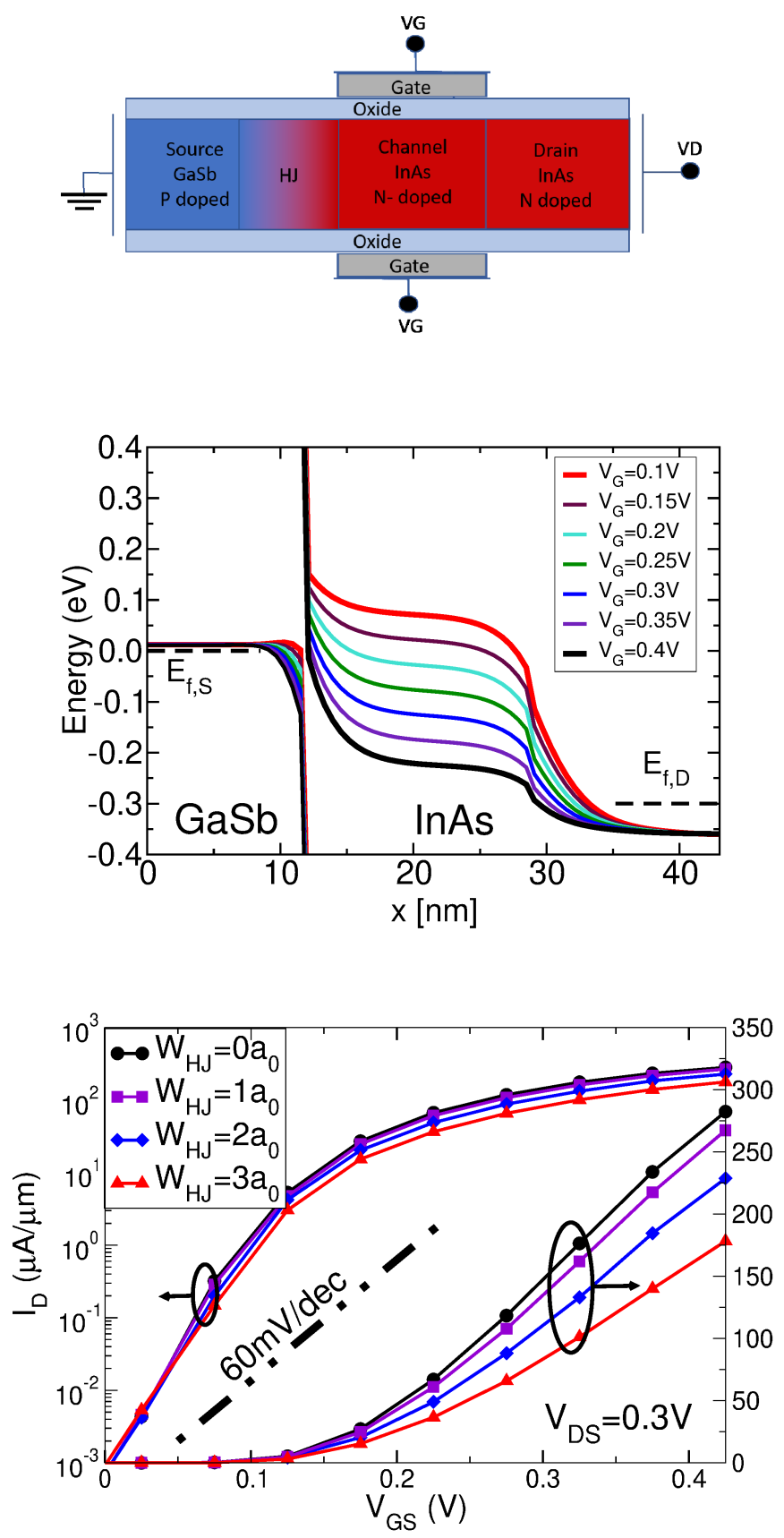

Fig. 6. (Top) Sketch of the simulated $n$-type HJ TFET. (Middle) Conduction and valence band profile at different $V_{G S}$ and Fermi level in the source region $E_{f, S}$ and drain region $E_{f, D}$ for a abrupt heterojunction GaSb-InAs $n$-type tunnel FET. (Bottom) Drain-current versus gate-voltage characteristics at $\mathrm{V}_{D S}=0.3 \mathrm{~V}$ for different widths, $W_{H J}$, of the heterojunction transition region. The metal gate workfunction was set to $4.625 \mathrm{eV}$.

In this work we used the pseudo-oxide EPM parameters proposed in [28], which result in a conduction and valence band discontinuity between InAs and the pseudo-oxide of respectively $3.8 \mathrm{eV}$ and $4.5 \mathrm{eV}$.

A sketch of the $n$-type heterojunction Tunnel FET is shown in Fig. 6 and the main device parameters are reported in Tab. II. Fig. 6 also reports the current versus gate voltage characteristics of the TFET for different widths, $W_{H J}$, of the GaSb-InAs heterojunction and for $\mathrm{V}_{D S}=0.3 \mathrm{~V}$. For both the 


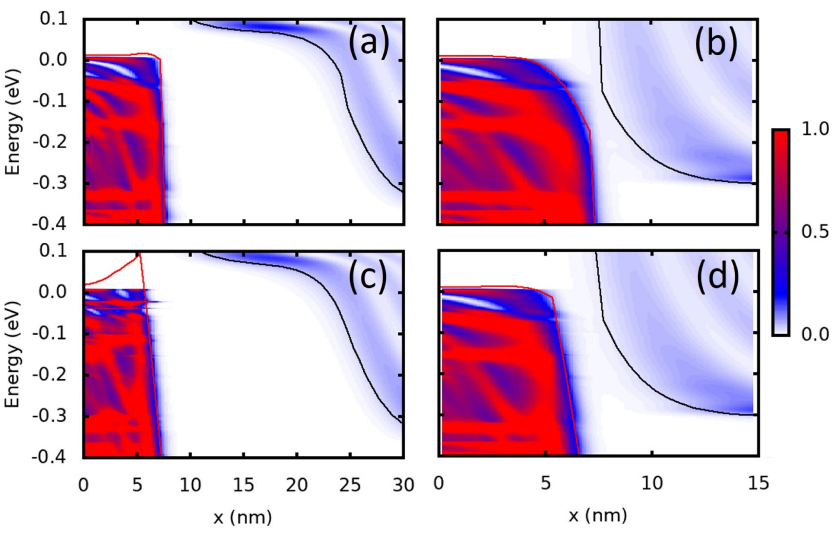

Fig. 7. Local density of states, LDOS, for the T-FET simulated in Fig.6. (a),(c) report the LDOS in the off state for $V_{G S}=0.0 \mathrm{~V}$ and $V_{D S}=0.3 \mathrm{~V}$; (b),(d) report the LDOS in the on state for $V_{G S}=0.4 \mathrm{~V}$ and $V_{D S}=0.3 \mathrm{~V}$. Plots in (a) and (b) correspond to an abrupt heterojunction (i.e. $W_{H J}=0$ ), while plots in (c) and (d) correspond to $W_{H J}=3 a_{0}$.

$n$ - and $p$-type TFET the metal gate workfunction was adjusted so that the off current (i.e. the $I_{D}$ at $\mathrm{V}_{G S}=0 \mathrm{~V}$ and $\mathrm{V}_{D S}$ $=0.3 \mathrm{~V}$ ) is approximately $1 \mathrm{nA} / \mu \mathrm{m}$. The transistor exhibits a very steep sub-threshold region with a sub-threshold swing below $60 \mathrm{mV} / \mathrm{dec}$, which is an expected result for a device with no defects and with an semiconductor-oxide interface free of traps and microscopic roughness [38], [39].

Quite interestingly the width $W_{H J}$ of the GaSb-InAs heterojunction has no sizeable influence on the subthreshold current and swing but it does affect the on current, which is in fact degraded when the transition region is wider. Fig. 7 shows that the markedly different influence of $W_{H J}$ on the off and on current is again related to its modulation of the band-to-bandtunnelling path. In the off-state, in fact, the impact of $W_{H J}$ on the relatively long BTBT path is only modest, whereas in the on state the relative importance of $W_{H J}$ is larger because the tunnelling path is much shorter.

TABLE III

DEVICE PARAMETERS FOR THE HETEROJUNCTION $p$-TYPE TUNNEL FET SIMULATED IN FIG. 8.

\begin{tabular}{|c|c|c|c|c|c|}
\hline \hline & $\begin{array}{c}T_{c h} \\
{[\mathrm{~nm}]}\end{array}$ & $\begin{array}{c}L_{G} \\
{[\mathrm{~nm}]}\end{array}$ & $\begin{array}{c}L_{S / D} \\
{[\mathrm{~nm}]}\end{array}$ & $\begin{array}{c}N_{A} \\
{\left[\mathrm{~cm}^{-3}\right]}\end{array}$ & $\begin{array}{c}N_{D} \\
{\left[\mathrm{~cm}^{-3}\right]}\end{array}$ \\
\hline InAs/GaSb & 3.7 & 16 & 14 & $5 \times 10^{19}$ & $2 \times 10^{19}$ \\
\hline
\end{tabular}

By leveraging the inherently full-band nature of our EPM based modelling approach, we also investigate a $p$-type heterojunction Tunnel FET, whose sketch is shown in Fig. 8 and whose parameters are reported in Tab. III. Due to the fairly low conduction band density of states of InAs, the source doping $N_{D}=2 \times 10^{19} \mathrm{~cm}^{-3}$ is large enough to induce a strongly degenerate electron gas in the source. Such a doping concentration is fairly consistent with the value recommended in Ref. [40], which was the outcome of an optimization of the tunnelling junction operation. The current-voltage characteristics in Fig. 8 show that the $p$-type Tunnel FET can hardly achieve a sub-threshold swing below $60 \mathrm{mV} / \mathrm{dec}$, with a degraded minimum swing compared to the $n$-type counterpart
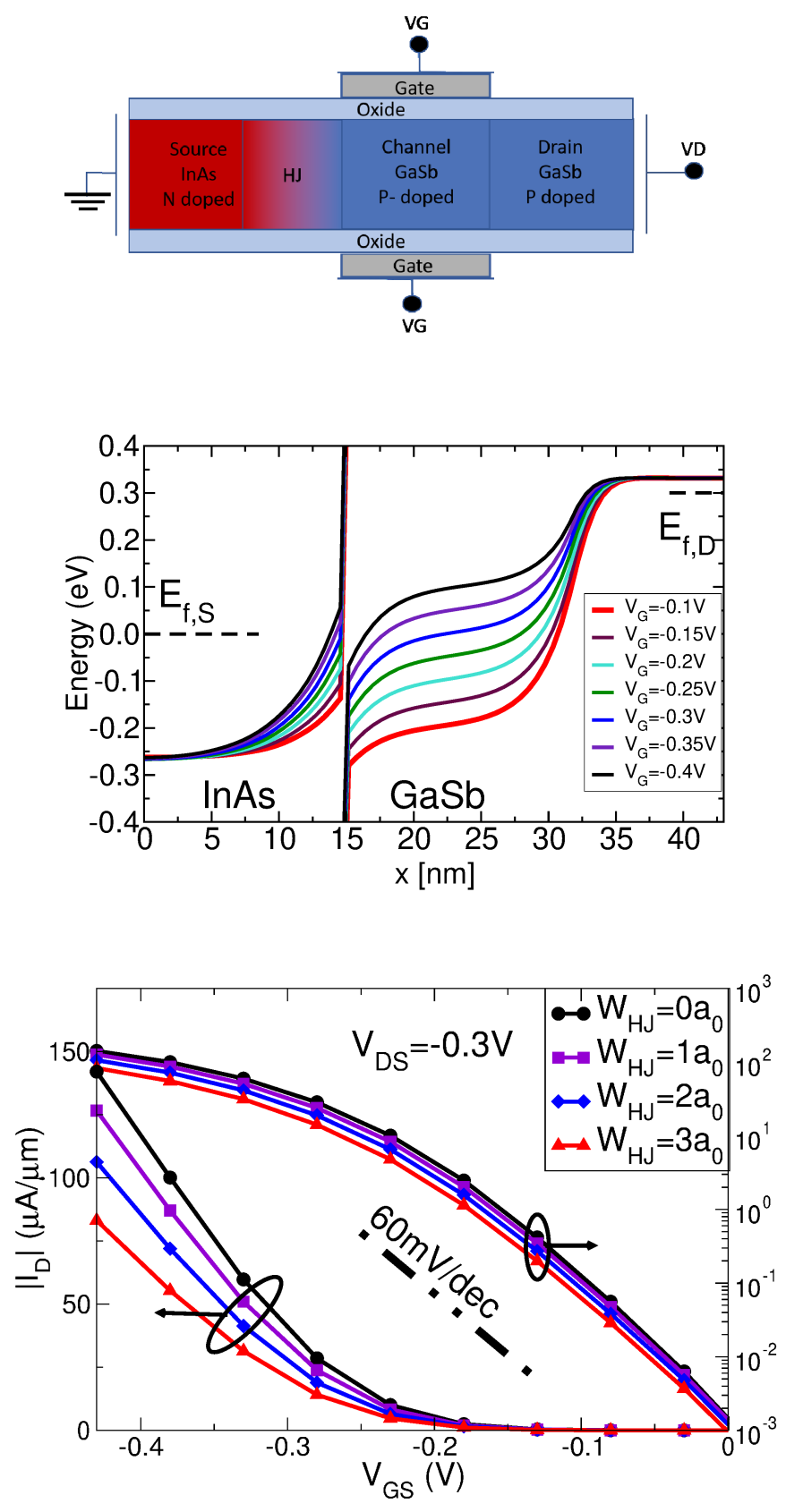

Fig. 8. (Top) Sketch of the simulated $p$-type HJ TFET. (Middle) Conduction and valence band profile at different $V_{G S}$ for a abrupt HJ GaSb-InAs $p$ type tunnel FET. (Bottom) Drain-current versus gate-voltage characteristics for different widths, $W_{H J}$, of the heterojunction transition region. The metal gate workfunction was set to $4.665 \mathrm{eV}$.

in Fig. 6. This result is not unexpected because the low density of states in the source region leads to a relatively large screening length of the electric field produced by the gate bias (compared to the $p$-type source of the $n$-type Tunnel FET) [41], which in turn degrades the sub-threshold swing [4].

As for the influence of the width $W_{H J}$ of the heterojunction transition region on the current-voltage characteristic of the device, the main features observed for the $n$-type Tunnel FET are confirmed. Namely, $W_{H J}$ hardly affects the subthreshold region, where the tunnelling distance is substantially larger than $W_{H J}$, whereas the on current of the device is 
progressively reduced for increasing $W_{H J}$. In the on state, in fact, a gradual transition region for the heterojunction results in a sizeable lengthening of the tunnelling path.

\section{CONCLUSION}

We have presented the implementation and application of a full band quantum transport methodology for nanoscale electron devices with heterojunctions. The model was developed in the framework of the empirical pseudopotentials method, and the heterojunctions were described according to a virtual crystal approximation, which allowed us to investigate the influence on the device characteristics of a gradual interface between the constituent materials.

We then leveraged the inherently full-band nature of the model and reported results for an $n$ - and a $p$-type, ultra-thin body heterojunction Tunnel FET, as well as for an Esaki diode.

The interface at the heterojunction was varied from the abrupt case to a more realistic transition width extending up to a few unit cells. The tunnelling current in the Esaki diode was found to be sensitive to the width of the heterojunction both in reverse bias and in the negative resistance region. In the GaSb-InAs Tunnel FETs the transition width affects the current only in the on-state. In the sub-threshold region, in fact, the effective tunnelling path is much larger than $W_{H J}$ and thus it is not appreciably modulated by heterojunction width.

The results of this work qualify our methodology based on the empirical pseudopotentials and the NEGF formalism as a viable approach for the modelling and design of homojunction and heterojunction nanoscale electron devices.

\section{REFERENCES}

[1] R. Kim, U. E. Avci, and I. A. Young, "Comprehensive Performance Benchmarking of III-V and Si nMOSFETs (Gate Length $=13 \mathrm{~nm}$ ) Considering Supply Voltage and OFF-Current," IEEE Trans. on Electron Devices, vol. 62, no. 3, pp. 713-721, 2015.

[2] D. Esseni, M. Pala, and T. Rollo, "Essential physics of the off-state current in nanoscale mosfets and tunnel fets," IEEE Trans. on Electron Devices, vol. 62, no. 9, pp. 3084-3091, 2015.

[3] A. Seabaugh and Q. Zhang, "Low-Voltage Tunnel Transistors for Beyond CMOS Logic," Proceedings of the IEEE, vol. 98, no. 12, pp. 2095 -2110 , dec. 2010.

[4] D. Esseni, M. Pala, P. Palestri, C. Alper, and T. Rollo, "A review of selected topics in physics based modeling for tunnel field-effect transistors," Semiconductor Science Technology, vol. 32, p. 083005 , 2017.

[5] D. Lizzit, P. Palestri, D. Esseni, A. Revelant, and L. Selmi, "Analysis of the Performance of n-Type FinFETs With Strained SiGe Channel," IEEE Trans. on Electron Devices, vol. 60, no. 6, pp. 1884-1891, 2013.

[6] T. Vasen, P. Ramvall, A. Afzalian, C. Thelander, K. A. Dick, M. Holland, G. Doornbos, S. W. Wang, R. Oxland, G. Vellianitis, M. J. H. van Dal, B. Duriez, J. R. Ramirez, R. Droopad, L. E. Wernersson, L. Samuelson, T. K. Chen, Y. C. Yeo, and M. Passlack, "InAs nanowire GAA nMOSFETs with 12-15 nm diameter," in IEEE Symposium on VLSI Technology - Technical Digest, June 2016, pp. 1-2.

[7] N. Loubet, T. Hook, P. Montanini, C. W. Yeung, S. Kanakasabapathy, M. Guillom, T. Yamashita, J. Zhang, X. Miao, J. Wang, A. Young, R. Chao, M. Kang, Z. Liu, S. Fan, B. Hamieh, S. Sieg, Y. Mignot, W. Xu, S. C. Seo, J. Yoo, S. Mochizuki, M. Sankarapandian, O. Kwon, A. Carr, A. Greene, Y. Park, J. Frougier, R. Galatage, R. Bao, J. Shearer, R. Conti, H. Song, D. Lee, D. Kong, Y. Xu, A. Arceo, Z. Bi, P. Xu, R. Muthinti, J. Li, R. Wong, D. Brown, P. Oldiges, R. Robison, J. Arnold, N. Felix, S. Skordas, J. Gaudiello, T. Standaert, H. Jagannathan, D. Corliss, M. H. Na, A. Knorr, T. Wu, D. Gupta, S. Lian, R. Divakaruni, T. Gow, C. Labelle, S. Lee, V. Paruchuri, H. Bu, and M. Khare, "Stacked nanosheet gate-all-around transistor to enable scaling beyond finfet," in 2017 Symposium on VLSI Technology, June 2017, pp. T230-T231.
[8] O. Badami, D. Lizzit, F. Driussi, P. Palestri, and D. Esseni, "Benchmarking of 3-d mosfet architectures: Focus on the impact of surface roughness and self-heating," IEEE Trans. on Electron Devices, pp. 3646 - 3653, Sept. 2018.

[9] J. S. Hamel, Y. T. Tang, and K. Osman, "Technological requirements for a lateral sige hbt technology including theoretical performance predictions relative to vertical sige hbts," IEEE Transactions on Electron Devices, vol. 49, no. 3, pp. 449-456, 2002.

[10] M. Schröter, T. Rosenbaum, P. Chevalier, B. Heinemann, S. P. Voinigescu, E. Preisler, J. Böck, and A. Mukherjee, "Sige hbt technology: Future trends and tcad-based roadmap," Proceedings of the IEEE, vol. 105, no. 6, pp. 1068-1086, 2017.

[11] U. E. Avci, D. H. Morris, and I. A. Young, "Tunnel field-effect transistors: Prospects and challenges," IEEE Journal of the Electron Devices Society, vol. 3, no. 3, pp. 88-95, 2015.

[12] S. Brocard, M. Pala, and D. Esseni, "Large On-Current Enhancement in Hetero-Junction Tunnel-FETs via Molar Fraction Grading," Electron Device Letters, IEEE, vol. 35, no. 2, pp. 184-186, Feb 2014.

[13] M. K. Hudait, Y. Lin, and S. A. Ringel, "Strain relaxation properties of inas metamorphic materials grown on inp substrates," Journal of Applied Physics, vol. 105, no. 6, p. 061643, 2009. [Online]. Available: https://doi.org/10.1063/1.3098232

[14] P. Guo, Y. Yang, Y. Cheng, G. Han, J. Pan, Ivana, Z. Zhang, H. Hu, Z. Xiang Shen, C. Kean Chia, and Y.-C. Yeo, "Tunneling field-effect transistor with ge/in0.53ga0.47as heterostructure as tunneling junction," Journal of Applied Physics, vol. 113, no. 9, p. 094502, 2013. [Online]. Available: https://doi.org/10.1063/1.4794010

[15] Y. Zhu, M. K. Hudait, D. K. Mohata, B. Rajamohanan, S. Datta, D. Lubyshev, J. M. Fastenau, and A. K. Liu, "Structural, morphological, and defect properties of metamorphic in0.7ga0.3as/gaas0.35sb0.65 ptype tunnel field effect transistor structure grown by molecular beam epitaxy," Journal of Vacuum Science \& Technology B, vol. 31, no. 4, p. 041203, 2013. [Online]. Available: https://doi.org/10.1116/1.4812793

[16] G. Kresse and J. Furthmüller, "Efficiency of ab-initio total energy calculations for metals and semiconductors using a plane-wave basis set," Computational Materials Science, vol. 6, no. 1, pp. 15 - 50, 1996. [Online]. Available: http://www.sciencedirect.com/science/article/pii/0927025696000080

[17] P. Giannozzi, S. Baroni, N. Bonini, M. Calandra, R. Car, C. Cavazzoni, D. Ceresoli, G. L. Chiarotti, M. Cococcioni, I. Dabo, A. Dal Corso, S. de Gironcoli, S. Fabris, G. Fratesi, R. Gebauer, U. Gerstmann, C. Gougoussis, A. Kokalj, M. Lazzeri, L. Martin-Samos, N. Marzari, F. Mauri, R. Mazzarello, S. Paolini, A. Pasquarello, L. Paulatto, C. Sbraccia, S. Scandolo, G. Sclauzero, A. P. Seitsonen, A. Smogunov, P. Umari, and R. M. Wentzcovitch, "Quantum espresso: a modular and open-source software project for quantum simulations of materials," Journal of Physics: Condensed Matter, vol. 21, no. 39, p. 395502 (19pp), 2009. [Online]. Available: http://www.quantum-espresso.org

[18] L.-W. Wang, A. Franceschetti, and A. Zunger, "Million-Atom Pseudopotential Calculation of $\Gamma-X$ Mixing in GaAs/AlAs Superlattices and Quantum Dots," Phys. Rev. Lett., vol. 78, no. 14, pp. 2819-2821, 1997.

[19] L.-W. Wang and A. Zunger, "Linear combination of bulk bands method for large-scale electronic structure calculations on strained nanostructures ," Phys. Rev. B, vol. 59, no. 24, pp. 15 806-15 818, 1999.

[20] F. Chirico, A. Di Carlo, and P. Lugli, "Efficient Self-consistent pseudopotential calculation of nanostructured devices," Phys. Rev. B, vol. 64, p. 045314, 2001.

[21] D. Esseni and P. Palestri, "Linear combination of bulk bands method for investigating the low-dimensional electron gas in nanostructured devices," Phys. Rev. B, vol. 72, no. 16, pp. 165342.1-165342.14, Oct 2005.

[22] J.-L. van der Steen, D. Esseni, P. Palestri, L. Selmi, and R.J.E. Hueting, "Validity of the parabolic Effective Mass Approximation in silicon and germanium n-MOSFETs with different crystal orientations," IEEE Trans. on Electron Devices, vol. 54, no. 8, pp. 1843-1851, 2007.

[23] Xiang-Wei Jiang, Shu-Shen Li, Jian-Bai Xia, and Lin-Wang Wang, "Quantum mechanical simulation of electronic transport in nanostructured devices by efficient self-consistent pseudopotential calculation," Journal of Applied Physics, vol. 109, p. 054503, 2011. [Online]. Available: http://dx.doi.org/doi/10.1063/1.3208067

[24] J. Fang, W. G. Vandenberghe, B. Fu, and M. V. Fischetti, "Pseudopotential-based electron quantum transport: Theoretical formulation and application to nanometer-scale silicon nanowire transistors," Journal of Applied Physics, vol. 119, p. 035701, 2016. [Online]. Available: http://dx.doi.org/doi/10.1063/1.3208067

[25] J. Fang, S. Chen, W. G. Vandenberghe, B. Fu, and M. V. Fischetti, "Theoretical Study of Ballistic Transport in Silicon Nanowire and Graphene 
Nanoribbon Field-Effect Transistors Using Empirical Pseudopotentials," Electron Devices, IEEE Transactions on, vol. 64, no. 6, pp. 2758 - 2764, 2017.

[26] M. L. Van de Put, M. V. Fischetti, and W. G. Vandenberghe, "Scalable atomistic simulations of quantum electron transport using empirical pseudopotentials," arXiv preprint arXiv:1903.00548, 2019.

[27] M. G. Pala and D. Esseni, "Full-band quantum simulation of electron devices with the pseudopotential method: Theory, implementation, and applications," Phys. Rev. B, vol. 97, p. 125310, Mar 2018. [Online]. Available: https://link.aps.org/doi/10.1103/PhysRevB.97.125310

[28] M. Pala and D. Esseni, "Quantum transport models based on NEGF and empirical pseudopotentials for accurate modeling of nanoscale electron devices," Journal of Applied Physics, vol. 126, no. 5, p. 055703, Aug. 2019. [Online]. Available: https://hal.archives-ouvertes.fr/hal-02350925

[29] M. G. Pala, P. Giannozzi, and D. Esseni, "Unit cell restricted bloch functions basis for first-principle transport models: Theory and application," Phys. Rev. B, vol. 102, p. 045410, Jul 2020. [Online]. Available: https://link.aps.org/doi/10.1103/PhysRevB.102.045410

[30] J. R. Chelikowsky and M. L. Cohen, "Electronic structure of silicon," Phys. Rev. B, vol. 10, no. 12, pp. 5095-5107, Dec 1974.

[31] M. L. Cohen and J. R. Chelikowsky, Electron Structure and Optical Properties of Semiconductors. Springer Series in Solid-State Sciences. Springer-Verlag Berlin Heidelberg New York London Tokyo, 1988.

[32] L. Bellaiche and D. Vanderbilt, "Virtual crystal approximation revisited: Application to dielectric and piezoelectric properties of perovskites," Phys. Rev. B, vol. 61, pp. 7877-7882, Mar 2000. [Online]. Available: https://link.aps.org/doi/10.1103/PhysRevB.61.7877

[33] I. Vurgaftman, J. R. Meyer, and L. R. Ram-Mohan, "Band parameters for III-V compound semiconductors and their alloys," Journal of Applied Physics, vol. 89, no. 11, pp. 5815-5875, 2001.

[34] B. M. Borg, K. A. Dick, B. Ganjipour, M.-E. Pistol, L.-E. Wernersson, and C. Thelander, "Inas/gasb heterostructure nanowires for tunnel fieldeffect transistors," Nano letters, vol. 10, no. 10, p. 4080, 2010.

[35] P. Friedel, M. S. Hybertsen, and M. Schlüter, "Local empirical pseudopotential approach to the optical properties of si/ge superlattices," Phys. Rev. B, vol. 39, pp. 7974-7977, Apr 1989. [Online]. Available: https://link.aps.org/doi/10.1103/PhysRevB.39.7974

[36] M. P. Anantram, M. S. Lundstrom, and D. E. Nikonov, "Modeling of Nanoscale Devices," Proceedings of the IEEE, vol. 96, no. 9, pp. 15111550, Sept 2008.

[37] R. Venugopal, Z. Ren, S. Datta, and M. Lundstrom, "Simulating quantum transport in nanoscale transistors: Real versus mode-space approaches," J. Appl. Phys., vol. 92, no. 7, pp. 3730-3739, 2002.

[38] F. Conzatti, M. Pala, D. Esseni, E. Bano, and L. Selmi, "Strain-Induced Performance Improvements in InAs Nanowire Tunnel FETs," Electron Devices, IEEE Transactions on, vol. 59, no. 8, pp. 2085-2092, 2012.

[39] F. Conzatti, M. Pala, and D. Esseni, "Surface-roughness-induced variability in nanowire InAs tunnel FETs," IEEE Electron Device Lett., vol. 33, no. 6, pp. 806-808, 2012.

[40] W. G. Vandenberghe, A. S. Verhulst, K.-H. Kao, K. D. Meyer, B. Soree, W. Magnus, and G. Groeseneken, "A model determining optimal doping concentration and material's band gap of tunnel field-effect transistors," Applied Physics Letters, vol. 100, no. 19, pp. 193509,2012. Available: https://doi.org/10.1063/1.4714544

[41] D. Lizzit, D. Esseni, P. Palestri, P. Osgnach, and L. Selmi, "Performance Benchmarking and Effective Channel Length for Nanoscale InAs, In0.53Ga0.47As, and sSi n-MOSFETs," IEEE Trans. on Electron Devices, vol. 61, no. 99, pp. 2027 - 2034, June 2014. 